\title{
SISTEMA INFORMATIZADO À DECISÃO CLÍNICA EM ENFERMAGEM: UMA CONSTRUÇÃO E VALIDAÇÃO NA ONCOLOGIA
}

Gisele Martins Miranda², Luciana Martins da Rosa², Kátia Cilene Godinho Bertoncello1, Nen Nalú Alves das Mercês², Lúcia Nazareth Amante ${ }^{1}$ Ana Graziela Alvarez

Objetivo: Descrever a construção e a validação dos registros eletrônicos do processo de enfermagem em uma instituição oncológica. Metodologia: Estudo de desenvolvimento metodológico realizado em Santa Catarina, entre 2015 e 2018 , sustentado pela Teoria de Elaboração de Instrumental Psicológico. Os procedimentos teóricos incluíram estudo de revisão, oficinas de discussão e de capacitação com validação semântica por consenso entre enfermeiros do cenário do estudo. Nos procedimentos experimentais realizou-se teste piloto e concluiu-se a validação semântica. Resultados: Incluíram-se 62 enfermeiros. Histórico de Enfermagem construido incluiu cinco domínios, 90 itens, 568 subitens. Selecionou-se das Taxonomias da North American Nursing Diagnosis Association 132 Diagnósticos, 143 Resultado e 176 Intervenções de Enfermagem, alimentados no sistema de informação na forma interconectada. Para os conteúdos da Avaliação de Enfermagem utilizou-se a matriz de dados do instrumento Histórico de Enfermagem. Conclusão: Os cadastros interconectados construidos favorecem a tomada de decisão do enfermeiro e qualificam o cuidado.

Descritores: Informática em enfermagem; Estudos de validação; Processo de enfermagem; Oncologia; Registros Eletrônicos de Saúde.

\section{CLINICAL DECISION COMPUTERIZED SYSTEM IN NURSING: A CONSTRUCTION AND VALIDATION IN ONCOLOGY}

Objective: To describe the construction and validation of electronic records of the nursing process in an oncological institution. Methodology: A methodological development study carried out in Santa Catarina, between January and April 2019, supported by the Theory of Elaboration of Psychological Instrumental. Theoretical procedures included review study, discussion and training workshops with semantic validation by consensus among nurses in the study setting. In the experimental procedures a pilot test was performed and the semantic validation was concluded. Results: 62 nurses were included. Built Nursing Historic included five domains, 90 items, 568 subitems. From the North American Nursing Diagnosis Association Taxonomies, 132 Diagnoses, 143 Outcomes, and 176 Nursing Interventions were selected. Subsequently inserted into the management system in interconnected form. For the contents of the Nursing Evaluation, the data matrix of the Nursing Historic instrument was used.

Conclusion: The interconnected records built favor nurses' decision making and qualify care.

Descriptors: Nursing informatics; Validation studies; Nursing process; Oncology; Electronic Health Records.

\section{SISTEMA INFORMATIZADO A LA DECISIÓN CLÍNICA EN ENFERMERÍA: UNA CONSTRUCCIÓN Y VALIDACIÓN EN LA ONCOLOGÍA}

Objetivo: Describir la construcción y validación de registros electrónicos del proceso de enfermería en una institución de oncología. Metodología: Estudio de desarrollo metodológico realizado en Santa Catarina, entre 2015 y 2018, apoyado por la Teoría del Desarrollo de Instrumentos Psicológicos. Los procedimientos teóricos incluyeron estudio de revisión, talleres de discusión y capacitación con validación semántica por consenso entre las enfermeras en el entorno del estudio. En los procedimientos experimentales se realizó una prueba piloto y se concluyó la validación semántica. Resultados: Se incluyeron 62 enfermeras. Histórico de Enfermería construido incluyó cinco dominios, 90 artículos, 568 subpuntos. De las Taxonomías de la North American Nursing Diagnosis Association, se seleccionaron 132 Diagnósticos, 143 Resultados y 176 Intervenciones de enfermería, alimentados al sistema de información en forma interconectada. Para los contenidos de la Evaluación de Enfermería, se utilizó la matriz de datos del instrumento Histórico de Enfermería. Conclusión: Los registros interconectados creados favorecen la toma de decisiones de las enfermeras y califican la atención.

Descriptores: Informática aplicada a la enfermeira; Estudios de Validación; Proceso de enfermería; Oncología; Registros Electrónicos de Salud. 


\section{INTRODUÇÃO}

Os sistemas de informação no Processo de Enfermagem (PE) tem contribuído de forma positiva para os registros do cuidado e gestão. Estudos sobre a implementação de um sistema de informações nos documentos de enfermagem apontam que a qualidade dos registros de enfermagem melhora significativamente a segurança, reduz riscos e contribui com a eficiência do trabalho de enfermagem ${ }^{(1-3)}$.

No Centro de Pesquisas Oncológicas (CEPON), uma instituição de Santa Catarina (SC), a implantação do PE em prontuário físico ocorreu em 1997. O referencial teórico escolhido pelos enfermeiros para sustentar a Sistematização da Assistência de Enfermagem (SAE) foi a associação das Teorias de Orem e Peplau ${ }^{(4)}$. Das cinco etapas do PE, quatro foram implantadas, a etapa dos Diagnósticos, naquele momento, limitou-se à identificação dos problemas em saúde.

Entre 1997 e 2011, o CEPON ampliou sua estrutura e a atenção ofertada à população e o uso das taxonomias de enfermagem ganhou relevância. Somado a esses fatos, em 2011, o CEPON adotou um software com linguagem de programação Delphi - Sistema de Gestão em Saúde TASY.

Em 2015, a alta gerência do CEPON identificou a necessidade de informatização do $P E$ e necessidade de atendimento à Resolução 358/2009 do Conselho Federal de Enfermagem. Por conseguinte, a informatização dos registros eletrônicos do PE no CEPON vem sendo desenvolvida por um movimento crescente. A aproximação e a participação da enfermeira do CEPON responsável pela informatização do PE com Curso de Mestrado Profissional alicerçou cientificamente esse processo de construção e estimulou o compartilhamento da experiência no meio científico.

Portanto, este estudo objetiva descrever a construção e a validação dos registros eletrônicos do processo de enfermagem em uma instituição oncológica.

\section{METODOLOGIA}

\section{Tipo de estudo}

Estudo de desenvolvimento metodológico, para construção e validação de instrumentos para registro eletrônico do processo de enfermagem, sustentado pela Teoria de Elaboração de Instrumental Psicológico de Luiz Pasquali(5).

Os estudos de validação referem-se à propriedade de um instrumento medir/verificar o que se propõe. A confiabilidade e a validade são consideradas as principais propriedades de medida dos instrumentos construídos, que desempenham um importante papel na prática clínica e na avaliação de saúde ${ }^{(6)}$. Por este motivo, a Teoria de Elaboração de Instrumental Psicológico foi escolhida para a elaboração dos produtos de enfermagem aqui apresentados.

\section{Local do estudo}

CEPON é uma instituição referência em SC desde 1986, que disponibiliza atendimento hospitalar (75 leitos) e atendimento ambulatorial as pessoas com todos os tipos de câncer. Em 2018, realizou 107.447 atendimentos ambulatoriais, 4.087 internações e 8.217 atendimentos de pronto socorro. A equipe de enfermagem do CEPON se constitui de 84 enfermeiros e 201 técnicos de enfermagem.

\section{População do estudo}

Foram incluidos enfermeiros atuantes no CEPON, coordenadores e enfermeiros assistenciais dos setores ambulatoriais e de internação, com atuação de, no mínimo, um ano na instituição e enfermeira atuante no Setor de Informática (TI) do CEPON.

\section{Coleta e análise dos dados}

A coleta e análise dos dados no estudo de desenvolvimento metodológico configuram as etapas de construção e de validação dos instrumentos. Resultados parciais são obtidos no transcorrer do processo de construção, até finalização da validação.

Dentre os procedimentos teóricos e experimentais, Pasquali propõem seis passos para a construção e validação do constructo de interesse ${ }^{(5)}$. Os conceitos de cada passo e as estratégias adotadas neste estudo serão apresentados a seguir na forma descritiva. Dividiu-se a descrição em procedimentos teóricos realizados no primeiro e segundo momento da construção e procedimentos experimentais.

Pasquali(5) ainda sugere a realização de procedimentos analíticos, mas apesar dos mesmos terem sido realizados, considerando o volume de dados, esta etapa não será apresentada neste artigo.

\section{Procedimentos teóricos - primeiro momento}

A Teoria de Elaboração de Instrumental Psicológico aponta a necessidade de definição inicial de um problema para sua aplicação. O problema encontrado foi à ausência de registros eletrônicos nas cinco etapas do PE e a existência apenas de registros em campos descritivos no prontuário do paciente. Diante desta problemática, enfermeira, com competência em informática foi escolhida, pela Gerência de Enfermagem, para atuar na TI do CEPON e informatizar o PE - criação dos registros eletrônicos de enfermagem. 


\section{Passos para a construção dos procedimentos teóricos}

10 passo - Sistema psicológico: ocorre a definição das ideias dos conteúdos dos instrumentos que devem ser construidos5. Neste estudo, constituiu-se pela definição da necessidade da criação dos conteúdos para registro eletrônico das etapas do PE - um instrumento para o Histórico de Enfermagem voltado ao contexto da enfermagem oncológica e outro instrumento contendo os Diagnósticos, Resultados e Intervenções de Enfermagem.

Para que isso ocorresse, a enfermeira atuante na TI iniciou estudo para compreender os parâmetros e cadastros do PE no TASY, utilizando a base de testes e homologações de versão do sistema.

2o passo - Propriedade do sistema psicológico: são delimitados os aspectos específicos que se deseja estudar e para os quais se quer construir um instrumento de medida(4). Neste contexto, realizou-se revisão narrativa (incluiu 20 publicações), livros textos e artigos científicos indexados em diversas bases de dados, que apresentavam as temáticas PE, informatização do PE e taxonomias de enfermagem.

A revisão narrativa inspirou a ideia de como elaborar o instrumento com adequação aos campos dos cadastros do Tasy, que contêm campos de seleção simples, multisseleção e descrição e a necessidade de definir-se com os enfermeiros do CEPON qual linguagem padronizada seria adotada para os registros dos Diagnósticos, Resultados e Intervenções de Enfermagem.

30 passo - Dimensionalidade do atributo: obtêmse as respostas se o atributo é uma unidade semântica única ou uma síntese de componentes diferentes ou até independentes5. Para tanto, fez-se a análise da Teoria de Orem e de Peplau, em revisão narrativa (15 publicações incluidas) e definiu-se que a estrutura interna do instrumento seria multifatorial.

4 passo - Definição dos construtos: divide-se em definição constitutiva e operacional, a primeira é aquela quando o construto é definido através de outros construtos4. Neste estudo utilizou-se as Teorias de Orem $^{(7)}$ e Peplau( ${ }^{(8)}$ e os Padrões de Saúde Funcional((9) como constructos orientadores. A segunda sustenta a legitimidade da representação empírica comportamental ${ }^{(5)}$. Neste estudo, esta definição vinculou-se ao perfil oncológico dos pacientes em relação aos instrumentos que se pretendia construir. Sequencialmente, os conteúdos definidos foram listados e categorizados.

5ㅇ passo - Operacionalização do construto: dá-se pela construção dos itens que representarão o constructo(5). Assim, agruparam-se os conteúdos categorizados, formando a la versão do instrumento Histórico de
Enfermagem. Esta construção foi digitada em arquivo do Programa Word da Microsoft. Os cinco primeiros passos foram realizados pela enfermeira atuante na TI.

Oficina de capacitação e de discussão com os enfermeiros foi realizada com objetivo de revisão dos conceitos sobre o PE e apresentação do Tasy, com demonstração do preenchimento e funcionalidades dos registros no sistema. Ainda nesta oficina, iniciou-se a validação semântica por consenso (6으 passo apresentado a seguir), disponibilizando-se aos enfermeiros uma cópia da $l^{\text {ạ }}$ versão do instrumento Histórico de Enfermagem para que pudessem verificar se os conteúdos eram compreensíveis e aplicáveis no cuidado ao paciente oncológico. Participaram dessa primeira oficina 18 enfermeiros. As contribuições dos enfermeiros foram agrupadas analisadas, quando construiu-se a $2^{\underline{a}}$ versão do instrumento.

Segunda oficina foi organizada para apresentação das taxonomias de enfermagem: Classificação Internacional para a Prática de Enfermagem - CIPE®, North American Nursing Diagnosis Association (NANDA) - Diagnósticos de Enfermagem da NANDA-Internacional (NANDA-I), Classificação dos Resultados de Enfermagem (NOC) e Classificação das Intervenções de Enfermagem (NIC) (9-11). O objetivo desta oficina foi a definição da linguagem padronizada a ser adotada na instituição. Decidiu-se pela adoção das taxonomias da NANDA-I (NANDA NNN)(19-11). Nesta oficina participaram dez enfermeiros.

Sequencialmenterealizou-senova revisão narrativa, com o objetivo de identificar-se os Diagnósticos de Enfermagem mais utilizado no contexto oncológico (12 publicações incluídas). Posteriormente, realizou-se mapeamento cruzado(12) entre os conteúdos do instrumento Histórico de Enfermagem com as características definidoras e fatores relacionados e de risco da NANDA-I para eleição dos Diagnósticos que deveriam ser selecionados para cadastramento no Tasy. Os achados foram cruzados com os resultados obtidos estudo de revisão, o que culminou na seleção final dos Diagnósticos, esses cruzados $^{(12)}$ com as taxonomias NOC e NIC para seleção dos Resultados e Intervenções de Enfermagem para cadastro no sistema.

No desdobramento das atividades, construiuse uma planilha no Programa Word contemplando a seleção dos Diagnósticos, Resultados e Intervenções de Enfermagem ${ }^{(9-11)}$ e determinou-se os grupos de dados e de ligações entre os mesmos. Assim, construiu-se os conteúdos do segundo instrumento proposto na definição das ideias nos procedimentos teóricos.

Em posse dos conteúdos dos dois instrumentos construídos, iniciou-se o cadastramento dos conteúdos 
no Tasy. Após o cadastro no sistema foram ajustados os dados no banco de dados relacional e trabalhadas as configurações para a funcionalidade da ferramenta, ou seja, elaborou-se banco de conteúdos para registro do PE, sendo as quatro primeiras etapas construídas na forma inter-relacionadas no sistema informatizado (cadastros interconectados). Os cinco primeiros passos foram realizados em 2015

Esta estratégia permite que o sistema, após preenchimento do Histórico de Enfermagem pelo enfermeiro, gere sugestões de Diagnósticos, Resultados e Intervenções de Enfermagem, que devem ser confirmadas ou não pelos enfermeiros. A relação dos dados nos cadastros do sistema ocorre através de algoritmos em uma linguagem de programação.

6 passo - Análise teórica dos itens: constitui-se pela análise dos conteúdos por juízes com o intuito de estabelecer a compreensão dos itens (análise semântica) e a pertinência dos mesmos ao atributo que se pretende medir (análise de conteúdo - procedimentos analíticos não apresentados neste artigo(13). Neste estudo os juízes na análise semântica foram os enfermeiros do CEPON.

A validação semântica sobre os conteúdos dos instrumentos foi realizada por consenso entre os enfermeiros participantes do estudo e ocorreu em 2016 com o desenvolvimento de mais uma oficina de discussão e capacitação.

Assim, realizou-se a terceira oficina para apresentação aos enfermeiros dos registros eletrônicos no prontuário do paciente. Participaram desta oficina oito enfermeiros, que após a apresentação dos conteúdos discutiram os conteúdos apresentados e validaram por consenso a sequência dos conteúdos e layout dos registros eletrônicos que deveriam compor os instrumentos. Frente às recomendações construiu-se 3a versão do instrumento Histórico de Enfermagem. Em relação aos instrumentos contendo os Diagnósticos, Resultados e Intervenções de Enfermagem os conteúdos foram aceitos na totalidade. Os enfermeiros apontaram que como os mesmos foram selecionados das taxonomias da NANDA, validação semântica não se fazia necessária.

\section{Descrição dos procedimentos experimentais}

Os procedimentos experimentais abrangeram a aplicação do teste piloto (três meses). Participaram desta etapa oito enfermeiros atuantes na Central de Quimioterapia. Iniciou-se o teste piloto em 2016, com a realização de uma consulta de enfermagem por dia. Ao término de cada consulta, a enfermeira da TI, in loco, orientava e auxiliava os enfermeiros na realização dos registros eletrônicos.

Neste momento, era solicitado um feedback do enfermeiro em relação ao uso da ferramenta informatizada, as recomendações dos enfermeiros eram analisadas pela enfermeira atuante na TI e os ajustes solicitados incorporados aos instrumentos informatizados.

Ampliou-se o teste piloto (três meses) com 18 enfermeiros atuantes na unidade de internação Cuidados Paliativos e Ambulatório de Adultos Jovens, com capacitação prévia dos profissionais. Neste período, quarta oficina de capacitação foi desenvolvida, com 18 enfermeiros, abordando a temática exame físico, pois observou-se incompletude dos registros de enfermagem. A análise dos dados para verificação da incompletude foi realizada pelas ferramentas disponíveis no Tasy.

Ao término do teste piloto construiu-se a $4 \underline{a}$ versão do instrumento Histórico de Enfermagem, considerando as contribuições dos enfermeiros. Sequencialmente, deuse início a implantação nos demais setores institucionais, quando construiu-se instrumento para os registros da Avaliação de Enfermagem. Para embasar esta construção, utilizou-se a matriz de dados do instrumento Histórico de Enfermagem, definiram-se itens de preenchimento obrigatório e adotou-se os cadastros interconectados do sistema. Desde o término do teste piloto, os registros do PE foram liberados para uso de todos os enfermeiros e pequenos ajustes dos conteúdos foram sendo incluídos, conforme solicitação da equipe.

\section{Procedimentos teóricos - segundo momento}

$\mathrm{Na}$ análise constante da equipe sobre os registros eletrônicos na prática clínica, evidenciou-se a fragilidade nos constructos do instrumento Histórico de Enfermagem incluindo a sustentação teórica proposta por Orem e Peplau ${ }^{(7-8)}$.

Nesse momento foi consensuado com os enfermeiros o aperfeiçoamento dos propósitos da Fase de Orientação da Teoria de Peplau e os Requisitos de Autocuidado Universal, Desenvolvimentais e Desvio de Saúde de Orem $^{(7-8)}$ nos conteúdos do Histórico de Enfermagem e definiu-se a necessidade de validação analítica com experts nas Teorias de Orem e Peplau, externos ao CEPON.

Então, voltou-se à literatura para nova aproximação com as teorias norteadoras da SAE no CEPON. Este fazer configurou a realização de novos procedimentos teóricos, previstos por Pasquali5 que culminou na elaboração da 5a versão do instrumento submetida à validação analítica. 


\section{Cuidados éticos}

Respeitou-se os princípios éticos e legais para pesquisas com seres humanos. Apreciação ética registrada sob os números 02799618.1.0000.0121 (proponente) e 02799618.1.3001.5355 (co-participante).

\section{RESULTADOS}

Participaram deste estudo 36 enfermeiros nos procedimentos teóricos e 26 nos procedimentos experimentais.

Os procedimentos teóricos e experimentais resultaram na elaboração de cinco versões do instrumento, intitulado "Histórico de Enfermagem" e banco de dados contendo os Diagnósticos, Resultados e Intervenções, segundo taxonomias da $\mathrm{Nanda}^{(9-11)}$, cadastrados de forma interconectada no Tasy, ou seja, os registros do Histórico de Enfermagem sugerem Diagnósticos, Resultados e Intervenções de Enfermagem que devem ser confirmado ou não pelo enfermeiro em sua tomada de decisão. Os registros eletrônicos construidos no Tasy foram registrados no prontuário do paciente sob o título SAE.

A última versão do Histórico de Enfermagem constituise por cinco domínios de conteúdos, 90 itens e 568 subitens, com opção de registro eletrônico para complementação de informações, de acordo com a necessidade identificada por cada enfermeiro. Todos os domínios dos conteúdos foram intitulados, "Fase de Orientação do Processo de Enfermagem".

O primeiro domínio do instrumento "Requisitos de Autocuidado Universal e por Desvio de Saúde" abrangeu a entrevista de enfermagem. O segundo domínio "Requisitos de Autocuidado Desenvolvimentais" abrangeu conteúdos sobre o conhecimento para controle e tratamento das comorbidades e a tomada de decisão do paciente. O terceiro domínio "Déficit de Autocuidado" abrangeu conteúdos sobre a avaliação do autocuidado do paciente. O quarto domínio "Requisito de Autocuidado por Desvio de Saúde" apresentou os conteúdos para registro do exame clínico do paciente. O quinto domínio "Sistemas de Enfermagem" permite - registro da necessidade do sistema totalmente compensatório; sistema parcialmente compensatório; apoio-educativo e independe para o autocuidado do paciente, proposto por Orem.

Foram cadastrados no sistema 132 Diagnósticos (81 com foco no problema, 46 de risco, dois de promoção da saúde e três de síndrome), 143 Resultados e 176 Intervenções de Enfermagem.

\section{DISCUSSÃO}

Reestruturar o Histórico de Enfermagem, neste estudo, com o uso de referencial teórico já conhecido e aceito pela equipe de enfermagem facilitou a adesão dos enfermeiros aos registros eletrônicos do PE. A adoção das taxonomias da NANDA(9-11) trouxe cientificidade à tomada de decisão clínica. Destaca-se que a construção dos registros eletrônicos do PE a partir de bancos de dados incluindo cadastros interconectados qualificou e agilizou a tomada de decisão do enfermeiro e vem servindo de exemplo inclusive para outras instituições que procuram a TI do CEPON para orientação das estratégias que devem ser adotadas para construção semelhante. Esta produção vem também favorecendo a capacitação dos profissionais recém admitidos e estudantes de enfermagem.

Entende-se que os instrumentos construidos configuram proposições inovadoras, que trouxeram melhorias e visibilidade à enfermagem oncológica no cenário do estudo(14)

O uso da tecnologia para informatizar os registros do PE abre um leque de benefícios para o paciente/cuidador, profissionais de enfermagem e instituição de saúde. Estes benefícios abrangem: Facilidade no acesso das informações; Agilidade na tomada de decisão e no cuidado: Apoio 24 horas do Serviço de Informática; Segurança das informações e do cuidado; Disponibilidade de mais tempo para o paciente e familiar; Menor deslocamento do setor/local de trabalho; Economia de papel/impressos: Organização dos dados; e Acesso às informações globais do paciente de qualquer lugar da instituição(15).

Com o desenvolvimento tecnológico atual e previsto para o futuro, os enfermeiros devem ser capazes de resolver problemas diante de uma infinidade de situações e condições em meio de diferentes adversidades. $\mathrm{O}$ uso do prontuário eletrônico, sistemas da informação e liderança da equipe configuram características indispensáveis para a melhor gestão do cuidado(16).

Estudo aponta que o dimensionamento deficitário de enfermeiros nas instituições e a falta de capacitação vêm abrindo lacunas para a realização do PE, bem como o tempo dispendido para os registros eletrônicos ${ }^{(17)}$. No cenário do estudo também ocorreram queixas dos profissionais relacionadas à quantidade de conteúdos a serem preenchidos no PE informatizado. No entanto, a iniciativa do PE informatizado ter partido da alta gerência e a gestão dos registros de enfermagem por meio do Setor de TI, e ainda, porque o cenário do estudo trabalha com indicadores de qualidade e auditorias periódicas, favoreceu a implementação do PE informatizado. 
Ainda destaca-se que, a inclusão dos profissionais de enfermagem do cenário do estudo nos procedimentos teóricos e experimentais foi de extrema valia, pois é o saber desses enfermeiros que estão na assistência direta ao paciente que enriqueceu a definição dos conteúdos dos registros eletrônicos de enfermagem, tornando o uso desta tecnologia mais eficaz.

\section{Limitações do estudo}

Como limite do estudo, aponta-se a inexistência de mensuração dos Resultados de Enfermagem, pois o disponibilizado no sistema permite apenas a identificação dos Resultados, assim, configurando uma etapa que necessita ser executada posteriormente.

\section{Contribuições para a prática}

A divulgação científica do processo de construção e validação dos registros eletrônicos do PE do CEPON poderá nortear a implantação em outras instituições

\section{CONCLUSÃO}

A construção e a validação dos registros eletrônicos do processo de enfermagem no CEPON sustentada pela Teoria de Elaboração de Instrumental Psicológico permitiu a construção dos produtos de enfermagem desejados, ou seja, os registros eletrônicos do PE com cadastros interconectados construídos no Sistema de Gestão em Saúde - TASY.

Os resultados obtidos favoreceram a tomada de decisão dos enfermeiros, trouxeram inovação para o contexto oncológico e poderão inspirar novas construções em outros cenários.

O presente trabalho foi realizado com apoio da Coordenação de Aperfeiçoamento de Pessoal de Nivel Superior - Brasil (CAPES) e do Conselho Federal de Enfermagem (COFEN), Edital 27/2016.

\section{REFERÊNCIAS}

1. Schachner B, González ZA, Sommer JA, Recondo FJ, Gassino FD, Luna DR et al. Computerization of a nursing chart according to the nursing process. Stud Health Technol Inform [Internet]. 2016 [cited 2019 May 30];225:133-1337. Available from: https://www.ncbi.nlm.nih.gov/pubmed/27332177

2. Lee J, Lee YJ, Bae JY, Seo MJ. Registered nurses' clinical reasoning skills and reasoning process: a think-aloud study. Nurse Educ Today [Internet]. 2016 [cited 2019 May 30]:46:75-80. Available from: https://doi.org/10.1016/j.nedt.2016.08.017

3. Wang P. Zhang H, Li B, Lin K. Making patient risk visible: implementation of a nursing document information system to improve patient safety. Stud Health Technol Inform [Internet]. 2016 [cited 2019 May 30];225:8-12. Available from: https://www.ncbi.nlm.nih.gov/pubmed/27332152

4. Rosa LM, Mercês NNA, Marcelino SR, Radünz V. A consulta de enfermagem no cuidado à pessoa com câncer: contextualizando uma realidade. Cogitare Enferm [Internet]. 2007 [cited 2019 May 30]; 12(4):487-93. Available from: http://dx.doi.org/10.5380/ce.v12i4.10075

5. Pasquali L. Psicometria. Rev Esc Enferm USP [Internet]. 2009 [cited 2019 May 30]; 43(Esp):992-9 Available from: http://www.scielo.br/pdf/reeusp/v43nspe/aO2v43ns.pdf

6. Souza AC, Alexandre NMC, Guirardello EB. Propriedades psicométricas na avaliação de instrumentos: avaliação da confiabilidade e da validade. Epidemiol. Serv. Saude [Internet]. 2017 [cited 2019 May 31]; 26(3):64959. Available from: http://scielo.iec.gov.br/pdf/ess/v26n3/2237-9622ess-26-03-00649.pdf

7. Taylor SG, Orem DE. Self-care deficit theory of nursing. In: Tomey AM Alligood MR, editors. Nursing Theorists and Their Work. Missouri: Mosby Elsevier; 2006.

8. Hagerty TA, Samuels W, Norcini-Pala A, Gigliotti E. Peplau's Theory of Interpersonal Relations: An Alternate Factor Structure for Patient Experience Data? Nurs Sci Q [Internet]. 2017 [cited 2019 May 30];30(2):160-167 Available from: doi: 10.1177/0894318417693286

9. NANDA International. Diagnósticos de enfermagem da NANDA: defini- ções e classificação 2015-2017; T. Heather Herdman, Shigemi Kamitsuru, organização; Regina Machado Garcez, tradução; Alba Lucia Bottura Leite de Barros, et al., revisão técnica. Porto Alegre: Artmed; 2015.

10. Bulechek GM, Butcher HK, Dochterman J, Wagner CM. NIC Classificação das Intervenções de Enfermagem. Tradução de Denise Costa Rodrigues. 6 ed. Rio de Janeiro: Elsevier: 2016.

11. Johnson M, Moorhead S, Maas ML, Swanson E. NOC Classificação dos Resultados de Enfermagem: mensuração dos resultados em saúde. Alba Lucia Bottura Leite de Barros, organização; Alcir Fernandes, et al., tradução. 5 ed. Rio de Janeiro: Elsevier; 2016.

12. Frauenfelder F, van Achterberg T, Needham I, Muller Staub M.. Nursing diagnoses in inpatient psychiatry. Int J Nurs Knowl [Internet]. 2016 [cited 2019 May 30]; 27( 1), 24-34. Available from: https://doi.org/10.1111/20473095.12068

13. Pasquali L. Psicometria: Teoria dos testes na Psicologia e na Educação. 5 ed. Petrópolis, RJ: Vozes; 2013

14. Silva MCN. Sistematização da assistência de enfermagem: desafio para a prática profissional. Enferm. foco (Brasilia) [Internet]. 2017 [cited 2019 May 30]; 8(3): Available from: http://revista.cofen.gov.br/index.php/ enfermagem/article/view/1534

15. Toney-Butler TJ, Thayer JM. Nursing Process [Updated 2019 Jul 30]. In: StatPearls [Internet]. Treasure Island (FL): StatPearls Publishing; 2019 [cited 2019 May 30]. Available from: https://www.ncbi.nlm.nih.gov/books/ NBK499937/

16. Costa C, Linch GFC. Implementation of electronic records related to nursing diagnoses. Int J Nurs Knowl [Internet]. 2018 [cited 2019 May 30]:00(0):1-9. Available from: https://doi.org/10.1111/2047-3095.12219

17. Andrade PME, Rocha ESB, Amorim SMR, Costa AMA da, Oliveira TAC de, Nolêto LL, et al. Sistematização da assistência de enfermagem: vantagens e dificuldades na sua aplicação sob a ótica de enfermeiros. Revista Eletrônica Acervo Saúde [Internet]. 2019[cited 2019 May 30]; 11(8):e588. Available from: https://doi.org/10.25248/reas.e588.2019 